\title{
Current correlations of Cooper-pair tunneling into a quantum Hall system
}

\author{
Andreas B. Michelsen $\odot,^{1,2}$ Thomas L. Schmidt, ${ }^{1}$ and Edvin G. Idrisov $\oplus^{1}$ \\ ${ }^{1}$ Department of Physics and Materials Science, University of Luxembourg, L-1511 Luxembourg, Luxembourg \\ ${ }^{2}$ SUPA, School of Physics and Astronomy, University of St Andrews, North Haugh, St Andrews KY16 9SS, United Kingdom
}

(Received 3 May 2020; revised 7 August 2020; accepted 17 August 2020; published 1 September 2020)

\begin{abstract}
We study Cooper-pair transport through a quantum point contact between a superconductor and a quantum Hall edge state at integer and fractional filling factors. We calculate the tunneling current and its finite-frequency noise to the leading order in the tunneling amplitude for $\mathrm{dc}$ and ac bias voltage in the limit of low temperatures. At zero temperature and in the case of tunneling into a single edge channel both the conductance and differential shot noise vanish as a result of the Pauli exclusion principle. In contrast, in the presence of two edge channels, this Pauli blockade is softened and a nonzero conductance and shot noise are revealed.
\end{abstract}

DOI: 10.1103/PhysRevB.102.125402

\section{INTRODUCTION}

The quantum Hall $(\mathrm{QH})$ effect $[1,2]$ is one of the most important effects of modern mesoscopic physics. Its main observable feature is the precise quantization of the Hall conductance to the value $G_{H}=v e^{2} / h$, where $v$ is the so-called filling factor. In a two-dimensional electron gas (2DEG) at integer $(v \in \mathbb{N})$ or certain fractional $[v=1 /(2 n+1)$ where $n \in \mathbb{N}$ ] filling factors, electron transport occurs through onedimensional (1D) channels located close at the edges of the sample [3]. The electron motion in these 1D channels is chiral, i.e., the electrons propagate in one direction with a speed of the order of $10^{4}$ to $10^{6} \mathrm{~m} / \mathrm{s} \mathrm{[4-6].} \mathrm{Electrons} \mathrm{in} \mathrm{such} \mathrm{edge}$ channels propagate ballistically without backscattering, in a way similar to photons in wave guides. This analogy has led to the emergence of the field of electron quantum optics which aims to realize quantum-optics-type experiments with electrons [7].

Recent progress in experimental techniques at the nanoscale has allowed experimentalists to create hybrid mesoscopic systems where $\mathrm{QH}$ edge states are coupled to other edge states [7-9], to quantum dots [6,10-13], to Ohmic contacts [14-17], or to superconductors [18-22]. This development has provided a successful platform to study some of the fundamental questions of mesoscopic physics, such as phase coherence [23-29], charge [14,30-32] and heat quantization [16,33], equilibration [34-39], and entanglement $[9,40]$. A particularly important setup for studying the transport properties of hybrid mesoscopic systems is based on $\mathrm{QH}$ edge states coupled to a metal via a quantum point contact (QPC), a narrow region between two electrically conducting systems. Such QPCs allow for tunneling experiments in the presence of an applied dc or ac bias voltage. In particular, the current and shot noise through a QPC connecting a $\mathrm{QH}$ edge state have been investigated in many experiments [7]. These experiments have made it possible to study the crossover from Fermi liquid to non-Fermi liquid phases in the $I-V$ (current-voltage) characteristics and in the corresponding noise measurements.
To study the transport in mesoscopic devices based on $\mathrm{QH}$ edge states, the low-energy effective theory developed by Wen is commonly used [41]. This bosonization approach shows that fractional edge states of the Laughlin series $[v=1 /(2 n+$ $1)$ ] can be modeled as Luttinger liquids with Luttinger parameter $K=v$. This theory has allowed the interpretation of the experimental data [7,9] obtained for transport properties of 1D chiral edge states. Moreover, the tunneling current and conductance, as well as the zero-frequency and finite-frequency nonequilibrium noise between edge states, were studied theoretically [42-60].

In these works, it was already shown that the typical behavior of the tunneling conductance of Laughlin fractional $\mathrm{QH}$ chiral edge states at low temperatures follows a power law, i.e., $G(T) \propto T^{2 g-2}$, where $T$ is the temperature and the parameter $g$ is equal to $v$ or $1 / v$ depending on the geometry of the QPC. Additionally it was shown that the behavior of the dc $I-V$ characteristic at zero temperature, low bias, and $g \neq 1$ is non-Ohmic, $I_{d c}(V) \propto V^{2 g-1}$, which is associated with the non-Fermi (Luttinger) liquid phase. In the case of a time-dependent bias voltage, $\tilde{V}(t)=V_{0}+V_{1} \cos (\Omega t)$ with frequency $\Omega$ and amplitude $V_{1}$ in the periodic ac part, the dc component of the current was found to have the form $I_{d c}=\sum_{n} J_{n}^{2}\left(e^{*} V_{1} / \hbar \Omega\right)\left|e^{*} V_{0}+n \hbar \Omega\right|^{2 g-1}$, where $e^{*}$ is the effective charge of the tunneling particle, $J_{n}\left(e^{*} V_{1} / \hbar \Omega\right)$ gives the Bessel function of the first kind, and $n$ is an integer number. Apart from the $I-V$ characteristic, the study of the zero- and finite-frequency noise in these references revealed a power-law dependence of the noise on the frequency at low temperatures. For instance, to the lowest order in the tunnel coupling, the finite-frequency symmetric noise at frequency $\omega$ is proportional to the sum of two terms $\left|\omega \pm \omega_{0}\right|^{2 g-1}$, which exhibit singularities at frequencies $\omega_{0}=e^{*} V / \hbar$ and $g<1 / 2$. In the case of a time-dependent bias voltage, the result gets modified similarly to the current to $\left|\omega \pm\left(\omega_{0}+n \Omega\right)\right|^{2 g-1}$, and again exhibits singularities at certain frequencies. The noise thus provides one of the most straightforward methods to measure the effective charge $e^{*}$ of tunneling Laughlin quasiparticles [7]. 
In the recent past, it has become possible to investigate such transport problems not only between identical ballistic chiral QH states but also between distinct systems, such as QH edge states and superconductors, both theoretically [61-70] and experimentally $[19,20,22,71-74]$. This line of research is particularly relevant for the creation of parafermion bound states, non-Abelian quasiparticles with potential application in topological quantum computation [75-84]. Motivated by this progress, we investigate the noise properties of the tunneling current between a superconductor and $\mathrm{QH}$ edge states at integer and Laughlin filling factors. We show that the previously demonstrated Pauli blockade [61] in the tunneling current at filling factor $v=1$ also manifests itself in shotnoise experiments. We expect that one can investigate shot noise and finite-frequency noise experimentally, as was done in Refs. [7] and [85] where the authors measured the dependence of noise on temperature and applied bias.

We note that due to the magnetic field the $\mathrm{QH}$ edge state is spinless (spin polarized), which suppresses any induced correlations from an $s$-wave superconductor (SC). This suppression can be lifted by spin-orbit coupling, such as the Rashba spinorbit coupling inherent to the geometry of a 2DEG [70]. This is relevant when the $\mathrm{QH}$ material is, e.g., InAs, but for the popular choice of graphene this is relatively weak. It has been suggested [22] that the QH edge state can be considered to have effective spin-orbit coupling inherited through proximity with a superconductor with bulk [86] or surface [87] spin-orbit coupling.

The rest of this article is structured as follows. In Sec. II, we introduce the model of a QPC in the spirit of Ref. [61]. In Sec. III, we calculate the tunneling current and the conductance perturbatively for a finite dc bias, which we will need in the following section. In Sec. IV, we calculate the finite-frequency noise in the dc regime. Section V is devoted to the derivation of the tunneling current and the finite-frequency noise for a periodic ac bias voltage. Finally, we present our conclusions and some future perspectives in Sec. VI. Details of the calculations and additional information are presented in the Appendixes. Throughout the paper, we set $|e|=\hbar=k_{B}=1$.

\section{THEORETICAL MODEL OF A QUANTUM POINT CONTACT}

We start by introducing the Hamiltonian of a QPC between a QH edge state at filling factor $v$ and an $s$-wave superconductor (see Fig. 1). To describe this system theoretically, we use the phenomenological model presented in Ref. [61]. We consider a total Hamiltonian of the system consisting of a term describing the $\mathrm{QH}$ edge, a term describing the $\mathrm{SC}$, and a tunneling term:

$$
\hat{H}=\hat{H}_{\mathrm{QH}}+\hat{H}_{\mathrm{SC}}+\hat{H}_{T} .
$$

The exact form of the term $\hat{H}_{\mathrm{QH}}$ depends on the filling factor and the cases of integer and fractional states as well as of a pair of co-propagating states are presented in the next sections.

Tunneling between the superconductor and the edge state is a two-step process. A Cooper pair from the superconducting condensate first splits into two electrons with opposite spins in a singlet state, both of which tunnel into the $\mathrm{QH}$ system.
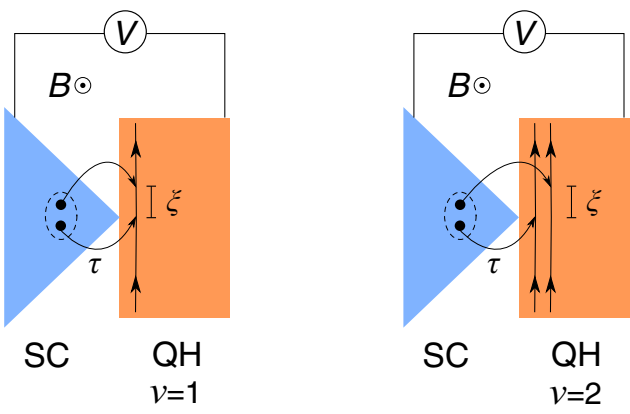

FIG. 1. Schematic representation of the system: a QPC with tunneling amplitude $\tau$ connects a superconductor (SC) to the chiral edge states of an integer quantum Hall $(\mathrm{QH})$ phase at filling factor $v$. At $v=1$ both electrons of the Cooper pair would have to occupy the same state, leading to a Pauli blockade, while at $v=2$ the electrons can enter different states. The bias is applied between the chiral edge channel and the superconductor.

However, since the edge state is spin polarized, a further spin-flip process, which can be brought about by spin-orbit coupling, is necessary to reach the final state which contains two electrons with the same spin propagating in the edge state. At temperatures much smaller than the superconducting gap, the Cooper pairs can be described as the mean value of the bosonic field $\hat{c}$ describing the superconducting condensate, $\Delta=\langle\hat{c}\rangle$. We assume that the Cooper pair tunneling happens at the point $x=0$, and we use this to build the tunneling Hamiltonian

$$
\begin{aligned}
\hat{H}_{T}= & \int d x d x^{\prime} t_{1}\left(x, x^{\prime}\right)\left(\hat{\psi}_{\uparrow}^{\dagger}(x) \hat{\psi}_{\downarrow}^{\dagger}\left(x^{\prime}\right) \hat{c}(x=0)+\text { H.c. }\right) \\
& +\int d x t_{2}(x)\left(\hat{\psi}_{\uparrow}^{\dagger}(x) \hat{\psi}_{\downarrow}(x)+\text { H.c. }\right),
\end{aligned}
$$

where $t_{1}$ and $t_{2}$ are tunneling and spin-flip amplitudes, respectively, and $\hat{\psi}_{\uparrow}(x)$ is the annihilation operator for a spin-up electron in the edge state at position $x$. If we consider the Hamiltonian perturbatively in $t_{1}$ and $t_{2}$, at second order we find the term

$$
\int d x d x^{\prime} \tau\left(x, x^{\prime}\right)\left[\hat{\psi}_{\uparrow}^{\dagger}(x) \hat{\psi}_{\uparrow}^{\dagger}\left(x^{\prime}\right) \hat{c}(x=0)+\text { H.c. }\right],
$$

where we have the effective tunneling parameter $\tau\left(x, x^{\prime}\right)=$ $t_{1}\left(x, x^{\prime}\right)\left[t_{2}(x)-t_{2}\left(x^{\prime}\right)\right]$. This term is the lowest-order term which includes both spin-flip and Cooper-pair tunneling in such a way as to remove the Cooper pair from the SC and create two spin-up electrons in the $\mathrm{QH}$ edge. Thus, at low energies this term will dominate the transport process across the interface, and we neglect all other terms. The term represents an effective $p$-wave pairing which is suppressed at short distances by the Pauli principle and vanishes exponentially at distances larger than the superconducting coherence length $\xi \propto v_{F} / \Delta$ [88], where $v_{F}$ is the Fermi velocity of the SC. This allows us to effectively approximate the term using a fixed distance $\xi$ between the electrons in the final state,

$$
\hat{H}_{T}^{\prime}=\tau \hat{\psi}_{\uparrow}^{\dagger}(x=\xi) \hat{\psi}_{\uparrow}^{\dagger}(x=0) \hat{c}(x=0)+\text { H.c. },
$$

without loss of qualitative generality [61]. From here on we suppress the spin index. 
In the following, we consider the effective Hamiltonian given by taking Hamiltonian (1) and replacing $\hat{H}_{T}$ with $\hat{H}_{T}^{\prime}$, which gives a complete description of the system under consideration. In the following, the relevant energy scales are assumed to be small compared to the Fermi energy, allowing us to use the effective low-energy theory to take into account the strong electron-electron interaction in edge states for the cases of filling factor $v=2$ and $v=1 /(2 n+1)(n \in \mathbb{N})$ $[41,42]$. The tunneling term (4) is considered perturbatively.

\section{TUNNELING CURRENT IN THE DC REGIME}

The operator for the tunneling current is given by $\hat{J}=$ $d \hat{N}_{\mathrm{QH}} / d t=i\left[\hat{H}, \hat{N}_{\mathrm{QH}}\right]$, where $\hat{N}_{\mathrm{QH}}=\int d x \hat{\psi}^{\dagger}(x) \hat{\psi}(x)$ is the electron number operator in the $\mathrm{QH}$ channel. It can be expressed as

$$
\hat{J}=2 i \tau \Delta\left(\hat{A}^{\dagger}-\hat{A}\right),
$$

where the operator $\hat{A}=\hat{\psi}(0) \hat{\psi}(\xi)$ consists of two fermionic fields. According to the real-time Keldysh approach the average tunneling current in the interaction picture is given by the expression

$$
I(t)=\left\langle\hat{U}^{\dagger}(t,-\infty) \hat{J}(t) \hat{U}(t,-\infty)\right\rangle,
$$

where the average is taken with respect to the dc biased ground state of $\mathrm{QH}$ edges and superconductor. The current becomes time independent once the system has reached a steady state. At the lowest order of tunneling coupling, the time-evolution operator is given by

$$
\hat{U}\left(t_{1}, t_{2}\right) \approx 1-i \int_{t_{2}}^{t_{1}} d t \hat{H}_{T}(t) .
$$

One then finds that the average tunneling current can be written in terms of a commutator of $A$ operators [89-91],

$$
I_{d c}(V)=2(\tau \Delta)^{2} \int_{-\infty}^{\infty} d t e^{2 i V t}\left\langle\left[\hat{A}^{\dagger}(t), \hat{A}(0)\right]\right\rangle_{0},
$$

where $V$ is the applied dc bias voltage. The average is taken with respect to the ground state of the uncoupled system, i.e., with respect to the equilibrium density matrix $\hat{\rho}_{0} \propto$ $\exp \left[-\left(\hat{H}_{\mathrm{QH}}+\hat{H}_{\mathrm{SC}}\right) / T\right]$, where $T$ is the temperature. The integrand of Eq. (8) only depends on one time variable due to time translation invariance in presence of $\mathrm{dc}$ bias. The prefactor 2 reflects the charge $2 e$ of the Cooper pairs. The perturbative result is valid as long as the tunneling current is small compared to the Hall current. Restoring the natural units, the Hall current is given by the relation $I_{H}=v e^{2} V / 2 \pi \hbar$.

\section{A. Filling factor $v=1$}

As an illustration of our approach based on Eq. (8), we first start by considering a system at filling factor $v=1$ and described by

$$
\hat{H}_{\mathrm{QH}}=-i v_{F} \int d x \hat{\psi}^{\dagger}(x) \partial_{x} \hat{\psi}(x) .
$$

Without loss of generality we consider right-moving fermions and focus on a positive applied dc bias voltage $V>0$. In the case of finite temperature $T$, an analytical continuation in the

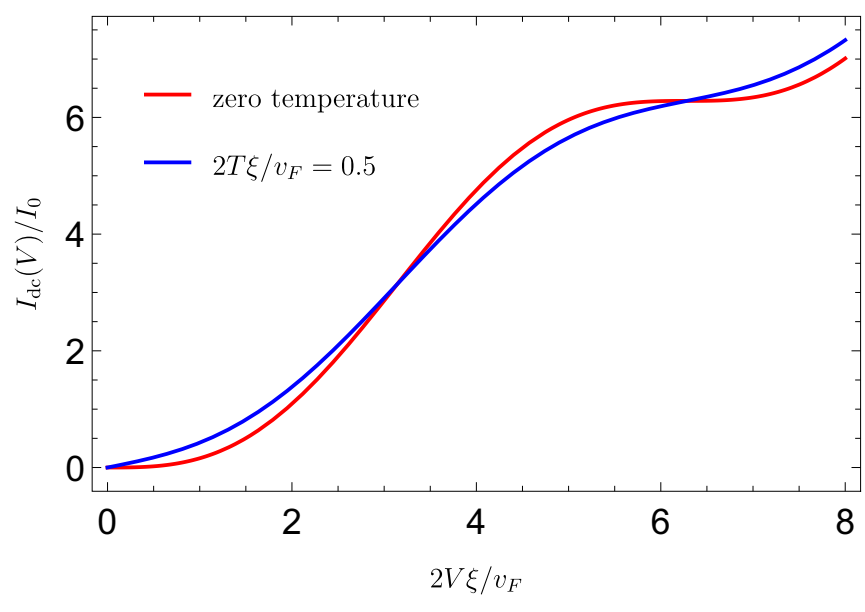

FIG. 2. The normalized tunneling current $I_{d c}(V) / I_{0}$ for filling factor $v=1$ oscillates with the dimensionless bias $2 V \xi / v_{F}$ around an Ohmic behavior, with nonzero temperature damping the oscillation [see Eqs. (10) and (13)].

complex plane is applied to Eq. (8). One finds the following result for the tunneling current:

$$
\begin{aligned}
I_{d c}(V) / I_{0}= & \frac{\xi T}{v_{F}} \sinh \left(\frac{V}{T}\right) \\
& \times\left[\mathcal{F}\left(0, \frac{V}{\pi T}\right)-\mathcal{F}\left(\frac{2 \pi \xi T}{v_{F}}, \frac{V}{\pi T}\right)\right],
\end{aligned}
$$

where $I_{0}=(\tau \Delta)^{2} / \pi v_{F} \xi$ is a normalization factor and the terms in square brackets are given by the integral

$$
\mathcal{F}(a, b)=\int_{-\infty}^{+\infty} \frac{\cos (b z) d z}{\cosh (a)+\cosh (z)} .
$$

Here one can check that the tunneling current vanishes at $V \rightarrow 0$ or $\xi \rightarrow 0$. In the general case, the result of Eq. (10) can be expressed in terms of hypergeometric functions. However, we are mainly interested in the regime of low temperature compared to the superconducting gap, $\xi T / v_{F} \ll 1$. Moreover, as we are mainly interested in the linear conductance, we also assume low voltages compared to the temperature scale, $V / T \ll 1$. In this case the result simplifies to $I_{d c}(V) \simeq$ $(4 / 3 \pi)\left(\tau \Delta / v_{F}\right)^{2} V\left(\pi \xi T / v_{F}\right)^{2}$.

A direct calculation of the conductance $G=\partial I_{d c}(V) / \partial V$ at $V \rightarrow 0$ from Eq. (10) gives

$$
G(T) / G_{0}=1-\frac{2 \pi \xi T / v_{F}}{\sinh \left(2 \pi \xi T / v_{F}\right)},
$$

where the normalization is equal to $G_{0}=2(\tau \Delta)^{2} / \pi v_{F}^{2}$. In the low-temperature limit $\xi T / v_{F} \ll 1$ we find that $G(T) / G_{0} \simeq$ $(2 / 3)\left(\pi \xi T / v_{F}\right)^{2}$.

Next, we discuss the results at zero temperature. Using Eq. (10), we obtain the expression for the tunneling current at $T=0$,

$$
I_{d c}(V) / I_{0}=\frac{2 V \xi}{v_{F}}\left[1-\frac{\sin \left(2 V \xi / v_{F}\right)}{2 V \xi / v_{F}}\right] .
$$

In the limit of small bias voltage $V \xi / v_{F} \ll 1$, we find nonOhmic behavior $I_{d c}(V) \propto(\tau \Delta)^{2} V^{3} \xi^{2} / v_{F}^{4}$, as shown in Fig. 2 . 
The oscillatory term is associated with the fact that the tunneling occurs at two points, separated by the superconducting coherence length, $\xi$. The linear QPC conductance associated with tunneling current is given by $G=\partial I_{d c}(V) / \partial V$ at $V \rightarrow$ 0 . The direct calculation gives $G=0$ at zero temperature. According to Ref. [61], the vanishing conductance and the non-Ohmic behavior of the tunneling current is related to the Pauli exclusion principle. At low energy scales, Pauli exclusion diminishes the effective density of states for electron-pair tunneling, namely, $\rho_{D O S} \propto\left(V \xi / v_{F}\right)^{2}$ at zero temperature and $\rho_{D O S} \propto\left(T \xi / v_{F}\right)^{2}$ at finite temperature. Physically this means that after the first electron has tunneled, the tunneling of a second electron is strongly suppressed up to times $t \sim \xi / v_{F}$.

\section{B. Filling factor $v=2$}

In this section, we consider the $\mathrm{QH}$ edge at filling factor $v=2$. First, we describe the noninteracting case. A pair of electrons from the superconductor can now tunnel simultaneously into two different edge channels [61], denoted by 1 and 2. To model this process, the electron operator in Eq. (5) can be represented as a superposition of independent fermionic fields $\hat{\psi}_{1,2}(x)$ as $\hat{\psi}=\sqrt{p} \hat{\psi}_{1}+\sqrt{1-p} \hat{\psi}_{2}$, where $p$ is the probability of an electron tunneling into edge state 1 , and $1-p$ is the probability of tunneling into edge state 2. To calculate the tunneling current (8) we need the two-point correlation functions $G_{j}\left(x_{1}-x_{2} ; t_{1}-\right.$ $\left.t_{2}\right)=\left\langle\hat{\psi}_{j}^{\dagger}\left(x_{1}, t_{1}\right) \hat{\psi}_{j}\left(x_{2}, t_{2}\right)\right\rangle_{0}$, where $j=1,2$ denotes the edge channel and for simplicity we assume both edge states to have the same Fermi velocity $v_{F}$. A difference in Fermi velocities could be absorbed into a redefinition of $p$.

At finite temperatures, similar steps as for filling factor $v=$ 1 lead to the following expression for the tunneling current:

$$
\begin{aligned}
I_{d c}(V) / I_{0}= & \frac{\xi T}{v_{F}} \sinh \left(\frac{V}{T}\right) \\
& \times\left[\mathcal{F}\left(0, \frac{V}{\pi T}\right)-\mathcal{N}(p, k \xi) \mathcal{F}\left(\frac{2 \pi \xi T}{v_{F}}, \frac{V}{\pi T}\right)\right],
\end{aligned}
$$

with $\mathcal{F}(a, b)$ defined as in Eq. (11). We have introduced the interference factor

$$
\mathcal{N}(p, k \xi)=1-2 p(1-p)[1-\cos (k \xi)],
$$

where $k=B l$ is the momentum difference between the two edge channels when separated by a length $l$ in a magnetic field of strength $B$. This reflects the inherent relationship between momentum and position of $\mathrm{QH}$ edge states [92], where taking the difference avoids all dependence on the choice of gauge. The result for the zero-bias conductance at finite temperature reads

$$
\frac{G(T)}{G_{0}}=1-\frac{2 \pi \xi T \mathcal{N}(p, k \xi)}{v_{F} \sinh \left(2 \pi \xi T / v_{F}\right)} .
$$

For $\xi T / v_{F} \ll 1$ we have $G(T) / G_{0} \simeq 1-\mathcal{N}(p, k \xi)+$ $(2 \mathcal{N}(p, k \xi) / 3)\left(\pi \xi T / v_{F}\right)^{2}$. The leading order generally does not vanish and does not depend on temperature. Physically this is due to a circumvention of the Pauli blockade by allowing the electrons to tunnel simultaneously into different channels.

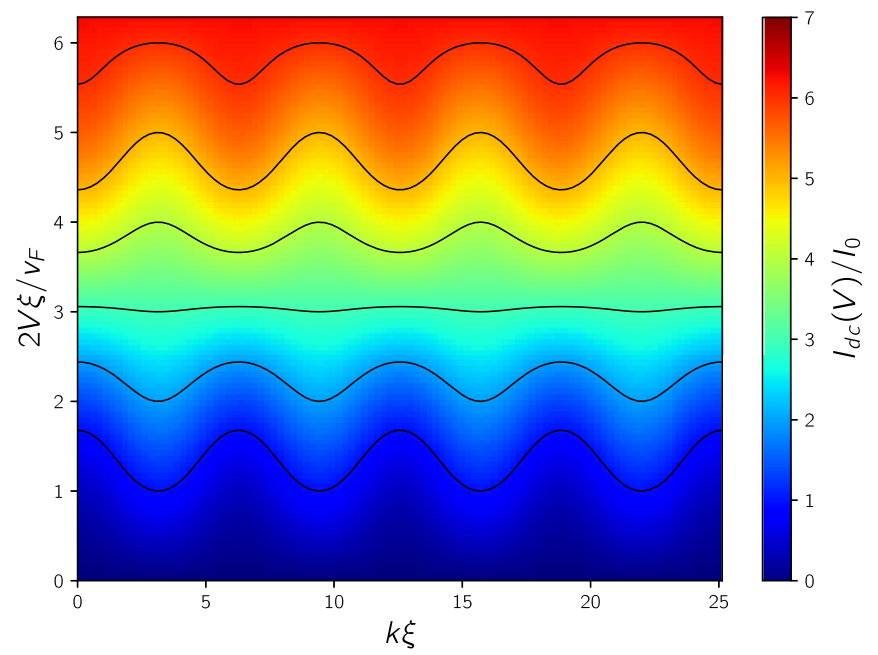

FIG. 3. At filling factor $v=2$, the normalized tunneling current oscillates both with the dimensionless bias $2 V \xi / v_{F}$ and the interference factor argument $k \xi$. These oscillations are shown at finite temperature, $2 \xi T / v_{F}=0.5$, and tunneling probability $p=0.5$ [see Eq. (14)]. Black lines indicate integer values of the current.

Employing Eq. (14), we get the result for the tunneling current at zero temperature,

$$
I_{d c}(V) / I_{0}=\frac{2 V \xi}{v_{F}}\left[1-\mathcal{N}(p, k \xi) \frac{\sin \left(2 V \xi / v_{F}\right)}{2 V \xi / v_{F}}\right] .
$$

As before, the current vanishes if either $V \rightarrow 0$ or $\xi \rightarrow 0$. For tunneling into a single edge state $(p=1$ or $p=0)$ one has $\mathcal{N}=1$ and thus recovers the result from Eq. (13). It is interesting to note that for arbitrary $p$, one can still have $\mathcal{N}=1$ if $\cos (k \xi)=1$, which is likely due to destructive interference of the two tunneling events. While most of the parameters in the cosine argument are material specific, and thus hard to vary experimentally, it should be possible to observe this recovery of the Pauli blockade by varying the $B$ field within an interval maintaining the $v=2$ filling factor.

In the limit $V \xi / v_{F} \ll 1$, we find that having two edge channels available and thus the possibility to avoid the Pauli blockade restores Ohmic behavior: $I_{d c}(V) \propto\left(\tau \Delta / v_{F}\right)^{2}(1-$ $\mathcal{N}) V$, whereas the subleading term is proportional to $(\tau \Delta)^{2} \mathcal{N} V^{3} \xi^{2} / v_{F}^{4}$. Equation (14) is shown in Fig. 3 for fixed finite temperature and tunneling probability. The oscillations with respect to the voltage are similar to those in Fig. 2, while the oscillations with respect to the interference factor argument $k \xi$ are related to the Pauli blockade. For a fixed applied bias, these Pauli blockade oscillations are peaked at $k \xi=$ $2 \pi n$, with $n \in \mathbb{N}$. At zero temperature these peaks become sharper, demonstrating a stronger blockade regime. Further, a straightforward calculation leads to the zero-temperature conductance $G(T=0) / G_{0}=1-\mathcal{N}(p, k \xi)$.

We now introduce electron-electron interactions both within a given edge state as well as between the two edge states. To study the effects of these interactions on the Pauli blockade, we start in the blockaded regime and thus assume that electrons only tunnel into one edge channel, corresponding to $p=1$ or $p=0$. To describe the edge states in the presence of interactions we use an effective 
field theory $[41,42]$. The edge-state excitations are then described as collective fluctuations of the charge density $\hat{\rho}_{j}(x)=$ $(1 / 2 \pi) \partial_{x} \hat{\phi}_{j}(x)$, where the index $j=1,2$ labels the edge state and $\hat{\phi}_{j}(x)$ is a bosonic field operator which satisfies the standard commutation relations $\left[\hat{\phi}_{i}(x), \hat{\phi}_{j}(y)\right]=i \pi \delta_{i j} \operatorname{sgn}(x-y)$. The Hamiltonian of the QH edge states is then given by

$$
\hat{H}_{\mathrm{QH}}=\frac{1}{2} \sum_{i j=1,2} \int d x \int d y \hat{\rho}_{i}(x) V_{i j}(x, y) \hat{\rho}_{j}(y),
$$

where the interaction kernel is given by $V_{i j}(x, y)=$ $\left(U+2 \pi v_{F} \delta_{i j}\right) \delta(x-y)$ with $U>0$ describing the screened Coulomb interaction. The Hamiltonian $\hat{H}_{\mathrm{QH}}$ can be diagonalized by the unitary transformation [89]

$$
\hat{\phi}_{1}=\frac{1}{\sqrt{2}}\left(\hat{\chi}_{1}+\hat{\chi}_{2}\right), \quad \hat{\phi}_{2}=\frac{1}{\sqrt{2}}\left(\hat{\chi}_{1}-\hat{\chi}_{2}\right),
$$

which conserves the bosonic commutation relations $\left[\hat{\chi}_{i}(x), \hat{\chi}_{j}(y)\right]=i \pi \delta_{i j} \operatorname{sgn}(x-y)$. Substituting these fields into the Hamiltonian (18) we obtain

$$
\hat{H}_{\mathrm{QH}}=\frac{1}{4 \pi} \sum_{j=1,2} v_{j} \int d x\left(\partial_{x} \hat{\chi}_{j}\right)^{2},
$$

which now contains a fast charge mode $(j=1)$ and a slow dipole mode $(j=2)$, with velocities $v_{1}=U / \pi+v_{F}$ and $v_{2}=v_{F}$, respectively. This bosonization procedure allows us to take into account electron-electron interactions with arbitrary strength explicitly and shows that the spectrum is split into two modes. Now, it is straightforward to calculate the four-point correlation functions using this diagonal Hamiltonian and the unitary transformation (19) (see Appendix B). Substituting the correlation functions from Eq. (B3) into Eq. (8), we get the following expression for the tunneling current at finite temperatures:

$$
\begin{aligned}
I_{d c}(V) / I_{0}= & \frac{v_{2}}{v_{1}} \frac{2 \xi T}{v_{2}} \sinh \left(\frac{V}{T}\right) \prod_{j=1,2} \sinh \left(\frac{\pi T \xi}{v_{j}}\right) \\
& \times \mathcal{J}\left(\frac{2 \pi T \xi}{v_{1}}, \frac{2 \pi T \xi}{v_{2}}, \frac{2 V}{\pi T}\right),
\end{aligned}
$$

where the last factor has the integral form

$$
\mathcal{J}\left(a_{1}, a_{2}, b\right)=\int_{-\infty}^{\infty} d y \frac{\cosh ^{-2}(y) \cos (b y)}{\prod_{i=1,2} \sqrt{\cosh (2 y)+\cosh \left(a_{i}\right)}} .
$$

At low temperatures $T \xi / v_{2} \ll 1$, the asymptotic form of the conductance is $G(T) / \mathcal{G}_{0} \simeq(2 / 3)\left(\pi \xi T / \sqrt{v_{1} v_{2}}\right)^{2}$, where $\mathcal{G}_{0}=2(\tau \Delta)^{2} / \pi v_{1} v_{2}$ is a normalization coefficient. At zero temperature and $V \xi / v_{2} \ll 1$ with $v_{1}>v_{2}$ we get $I_{d c}(V) \propto$ $(2 / 3 \pi)\left(V^{3} \xi^{2}\right) /\left(v_{1} v_{2}\right)^{2}$, resulting in vanishing zero-bias conductance. Thus we get the same result as in the case of filling factor $v=1$ in Eq. (13) at $V \xi / v_{F} \ll 1$. The Pauli blockade persists even with cross-channel interaction. As one can see, the interaction renormalizes the Fermi velocity, so to obtain the current at $T \rightarrow 0$ one has to change $v_{F}$ to $\sqrt{v_{1} v_{2}}$ in asymptotics of Eq. (13). The dependence of the tunneling current on the applied bias at different interaction parameters, $v_{2} / v_{1}$, is shown in Fig. 4. Here $v_{2} / v_{1}=1$ corresponds to the noninteracting case. One can see that the interaction parameter slightly decreases the magnitude of the tunneling

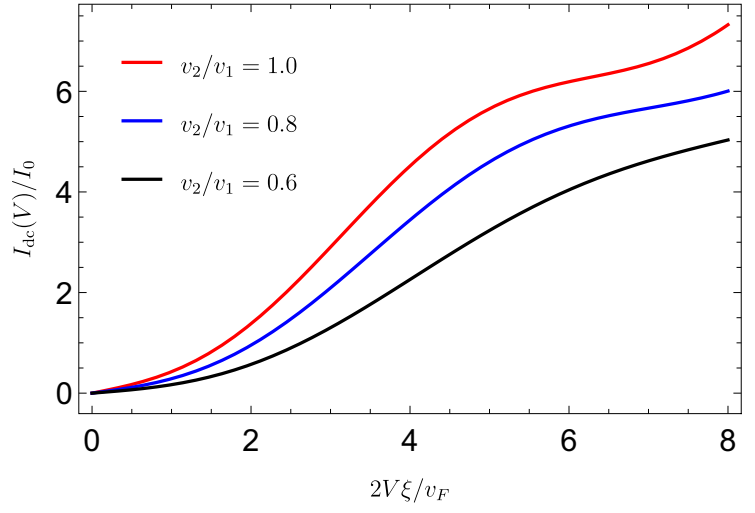

FIG. 4. When the electrons tunnel into two interacting QH edge states, we see a decrease in the magnitude of the normalized tunneling current, as well as longer oscillation periods with applied bias, for stronger interactions. The figure shows the case for finite temperature $2 \xi T / v_{F}=0.5$ with an interaction parameter $v_{2} / v_{1}$ which has $v_{2}=v_{F}$ [see Eq. (21)], and where $v_{2} / v_{1}=1.0$ is the noninteracting case.

current in comparison with the noninteracting regime, while the oscillation period is increased.

\section{Filling factor $v=1 /(2 n+1)$}

The fractional $\mathrm{QH}$ edge state with Laughlin filling factor $v=1 /(2 n+1), n \in \mathbb{N}$, consists of a single channel with a free bosonic field $\hat{\phi}(x)$ propagating with velocity $v$. The electron operator is given by the vertex operator $\hat{\psi}(x) \propto e^{i \hat{\phi}(x) / \sqrt{v}}$ $[41,42]$. We can then repeat the steps of the previous sections to get the tunneling current at finite temperature,

$$
\begin{aligned}
I_{d c}(V) / \tilde{I}_{0}= & 2^{1 / \nu} \frac{\xi T}{v}\left(\frac{r T}{2 v}\right)^{2 / v-2} \sinh ^{2 / v}\left(\frac{\pi \xi T}{v}\right) \\
& \times \sinh \left(\frac{V}{T}\right) \mathcal{Q}\left(\frac{2 \pi \xi T}{v}, \frac{2 V}{\pi T}\right),
\end{aligned}
$$

where $r$ is an ultraviolet cutoff, $\tilde{I}_{0}=(\tau \Delta)^{2} / \pi v \xi$ is the normalization coefficient, and we use the dimensionless integral

$$
\mathcal{Q}(a, b)=\int_{-\infty}^{\infty} d y \frac{\cosh ^{-2 / v}(y) \cos (b y)}{[\cosh (2 y)+\cosh (a)]^{1 / v}} .
$$

At $T \xi / v \ll 1$ we get the asymptotic behavior of the conductance,

$$
\frac{G(T)}{\tilde{G}_{0}} \simeq \frac{\sqrt{\pi}}{2} \frac{\Gamma(2 / v)}{\Gamma(1 / 2+2 / v)}\left(\frac{r T}{2 v}\right)^{2 / v-2}\left(\frac{\pi \xi T}{v}\right)^{2 / v},
$$

where $\Gamma(x)$ denotes the gamma function and $\tilde{G}_{0}=$ $2(\tau \Delta)^{2} / \pi v^{2}$. Further, at zero temperature and low voltages $V \xi / v \ll 1$, using Eq. (23), we find that the current has the form

$$
I_{d c}(V) / \tilde{I}_{0} \simeq \frac{2 \pi^{2} v \xi}{r^{2}}\left(\frac{2 r \xi}{\pi v^{2}}\right)^{2 / v} \frac{V^{4 / v-2}}{\Gamma(4 / v)} .
$$

Consequently, the conductance vanishes as in the case of filling factor $v=1$, i.e., $G=0$. This result can also be obtained from Eq. (25) at $T \rightarrow 0$. This is related to the power-law 
behavior of the tunneling current with respect to the applied voltage due to the positive integer power in Eq. (26). Even though we have tunneling between two effectively bosonic systems, the Pauli blockade persists and makes the QPC an insulator at zero bias. At filling factor $v=1$ the results of this section coincide with the results of Sec. III A.

\section{FINITE-FREQUENCY NOISE IN THE DC REGIME}

In this section, we consider the finite-frequency noise in the case of an applied dc voltage. The exact experimentally measurable current noise depends on the details of the setup, so we calculate the nonsymmetrized current correlation function from which other forms of noise, e.g., the symmetrized noise, can be obtained $[48,91,93,94]$. It is defined as

$$
S_{d c}(\omega, V)=\int_{-\infty}^{+\infty} d t e^{i \omega t}\langle\delta \hat{J}(t) \delta \hat{J}(0)\rangle
$$

where $\delta \hat{J}(t)=\hat{J}(t)-\langle\hat{J}(t)\rangle$ and the average is taken with respect to the dc biased ground state of the $\mathrm{QH}$ system and superconductor. Using the time translation invariance of the vertex operators (see Appendix C), the noise can be written to the lowest order of the tunneling coupling as

$$
S_{d c}(\omega, V)=g(\omega+2 V)+g(\omega-2 V),
$$

where the correlation function on the right is given by

$$
g(\omega)=4(\tau \Delta)^{2} \int_{-\infty}^{+\infty} d t e^{i \omega t}\left\langle\hat{A}^{\dagger}(t) \hat{A}(0)\right\rangle_{0} .
$$

It is worth pointing out that the shot noise at $\omega=0$ is determined by the anticommutator of $\hat{A}$ operators, in contrast to the tunneling current in Eq. (8), namely, $S_{d c}(0, V)=$ $4(\tau \Delta)^{2} \int_{-\infty}^{+\infty} d t e^{2 i V t}\left\langle\left\{A^{\dagger}(t), A(0)\right\}\right\rangle_{0}$ [91]. The noise can be symmetrized as the even combination of the two nonsymmetrized terms, $[S(\omega)+S(-\omega)] / 2$, and whether measuring the nonsymmetrized or the symmetrized noise is possible depends on the experimental detector [93].

\section{A. Filling factor $v=2$}

We start again by considering a system with positive bias voltage $V>0$, no interactions, and Cooper pairs tunneling simultaneously into both edge channels (see Sec. III B). Using Eq. (27) and the two-point correlation functions from Appendix A, the noise at finite temperature becomes

$$
\begin{aligned}
S_{d c}(\omega, V) / I_{0}= & \sum_{\sigma= \pm} \frac{\xi T}{v_{F}} \exp \left(\frac{\omega+2 \sigma V}{2 T}\right)\left[\mathcal{F}\left(0, \frac{\omega+2 \sigma V}{2 \pi T}\right)\right. \\
& \left.-\mathcal{N}(p, k \xi) \mathcal{F}\left(\frac{2 \pi \xi T}{v_{F}}, \frac{\omega+2 \sigma V}{2 \pi T}\right)\right],
\end{aligned}
$$

where $\mathcal{F}(a, b)$ is defined in Eq. (11) and the normalization coefficient $I_{0}$ is given after Eq. (10).

At zero temperature we can use Eq. (30) to obtain the expression

$$
\begin{aligned}
S_{d c}(\omega, V) / I_{0}= & \sum_{\sigma= \pm} 2 \theta\left(\omega \xi / v_{F}+2 \sigma V \xi / v_{F}\right)\left\{\frac{|\omega+2 \sigma V| \xi}{v_{F}}\right. \\
& \left.-\mathcal{N}(p, k \xi) \sin \left(\frac{|\omega+2 \sigma V| \xi}{v_{F}}\right)\right\}
\end{aligned}
$$

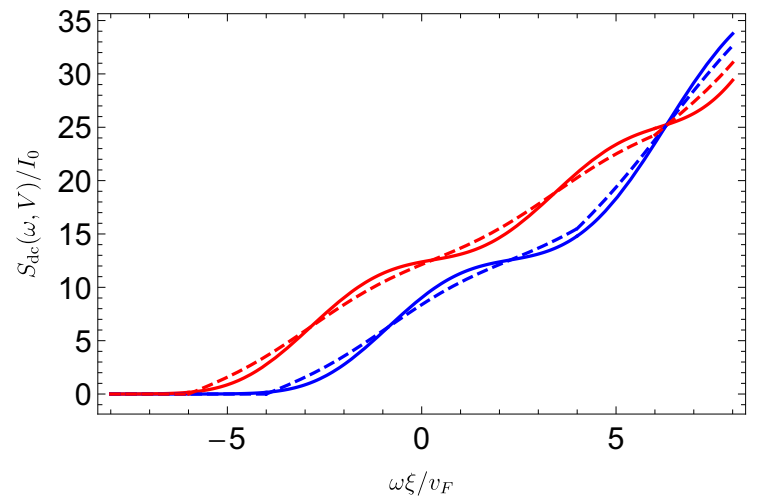

FIG. 5. The normalized finite-frequency nonsymmetrized noise of the dc tunneling current $S_{d c}(\omega, V) / I_{0}$ at filling factor $v=2$ as a function of the dimensionless frequency $\omega \xi / v_{F}$ at applied voltages $2 V \xi / v_{F}=4$ (blue lines) and $2 V \xi / v_{F}=6$ (red lines). Here, the solid lines correspond to finite temperature $2 T \xi / v_{F}=0.5$ and the dashed lines correspond to zero temperature. Notably, we find no resonances (singularities). We have $p=0.5$ and $k \xi=2 \pi / 3$. See Eqs. (30) and (31).

where $\mathcal{N}(p, k \xi)$ is given in Eq. (17) and $\theta(x)$ is the Heaviside step function. The dependence of noise on frequency at zero and finite temperatures is shown in Fig. 5. The oscillations are again related to the tunneling of electrons into two spatially separated points, $x=0$ and $x=\xi$. At small frequencies $0<\omega \ll 2 V$, the linear-frequency-dependent part appears in a subleading term, namely, $S_{d c}(\omega, V) / I_{0} \approx 2\left(2 V \xi / v_{F}-\right.$ $\left.\mathcal{N} \sin \left(2 V \xi / v_{F}\right)\right)+2\left(1-\mathcal{N} \cos \left(2 V \xi / v_{F}\right)\right)\left(\omega \xi / v_{F}\right)$. At large frequencies $\omega \gg 2 V>0$, the frequency-dependent part appears in the leading order, $S_{d c}(\omega, V) / I_{0} \approx 4 \omega \xi / v_{F}$. Furthermore, we calculate the derivative of the shot noise with respect to the applied bias at $V \rightarrow 0$ and get

$$
\left.G_{0}^{-1} \frac{\partial S_{d c}(0, V)}{\partial V}\right|_{V \rightarrow 0}=2[1-\mathcal{N}(p, k \xi)]
$$

where the prefactor $G_{0}$ on the left is given after Eq. (12). Here, as with the corresponding conductance, we see that at filling factor $v=2$ the Pauli blockade is lifted. At low temperatures $\xi T / v_{F} \ll 1$, the subleading correction to Eq. (32) is given by $(4 / 3) \mathcal{N}(p, k \xi)\left(\pi \xi T / v_{F}\right)^{2}$; i.e., the temperature independence is only to leading order.

Furthermore, using Eqs. (14) and (30), and $\mathcal{F}(a,-b)=$ $\mathcal{F}(a, b)$, one can see that the Fano factor has the well-known form [7]

$$
S_{d c}(0, V) / I_{d c}(V)=2 \operatorname{coth}\left(\frac{V}{T}\right) .
$$

Therefore, the current fluctuations satisfy a classical Poissonian shot-noise form [7]. At zero temperature, we have $\operatorname{coth}(V / T) \rightarrow 1$, so the Fano factor becomes $S_{d c}(0, V) / I_{d c}(V)=2$, which can be taken as an indication that the elementary charge carriers tunneling through the QPC are indeed charge- $2 e$ Cooper pairs.

The result for filling factor $v=1$ can be obtained setting $\mathcal{N}(p, k \xi)=1$ at $p=1$ or $p=0$. In particular, at low temperatures and bias voltage, $\xi T / v_{F} \ll 1$ and $V / T \ll 1$, we get

$$
S_{d c}(0, V) / I_{0} \simeq \frac{8 V \xi}{3 v_{F}}\left(\frac{\pi \xi T}{v_{F}}\right)^{2} .
$$


Thus, the right-hand side of Eq. (34) becomes proportional to the differential conductance (12) at $V \rightarrow 0$; i.e., $\partial S_{d c}(0, V) / \partial V \propto\left(\xi T / v_{F}\right)^{2}$.

Taking into account electron-electron interactions, assuming tunneling into only one channel, and repeating the steps leading to Eqs. (21) and (30) for finite temperatures yields the following result:

$$
\begin{aligned}
S_{d c}(\omega, V) / I_{0}= & \sum_{\sigma= \pm} \frac{v_{2}}{v_{1}} \frac{2 \xi T}{v_{2}} \exp \left(\frac{\omega+2 \sigma V}{2 T}\right) \\
& \times \prod_{i=1,2} \sinh \left(\frac{\pi T \xi}{v_{i}}\right) \\
& \times \mathcal{J}\left(\frac{2 \pi T \xi}{v_{1}}, \frac{2 \pi T \xi}{v_{2}}, \frac{\omega+2 \sigma V}{\pi T}\right),
\end{aligned}
$$

where $I_{0}$ is given in Eq. (21), and $\mathcal{J}$ is given in Eq. (22). Calculating the zero-frequency noise, one finds that the Fano factor is given by Eq. (33), as it is expected. It is worth mentioning here that at $V \rightarrow 0$, the differential shot noise vanishes.

At zero temperature, using Eq. (35), for the shot noise at $\omega=0$ and $V \xi / v_{1}, V \xi / v_{2} \ll 1$ we get

$$
S_{d c}(0, V) / I_{0} \simeq \frac{8 v_{2}^{2}}{3 v_{1}^{2}} \frac{V^{3} \xi^{3}}{v_{2}^{3}} .
$$

Direct calculations using the asymptotic result in Eq. (36) or the exact expression at $\omega=0$ in Eq. (35) give that $\partial S_{d c}(0, V) / \partial V=0$ at $V \rightarrow 0$, which is caused by the Pauli blockade.

\section{B. Filling factor $v=1 /(2 n+1)$}

Repeating the steps of the previous sections, in the case of finite temperature we get the following result for noise at fractional filling factors:

$$
\begin{aligned}
S_{d c}(\omega, V) / \tilde{I}_{0}= & \sum_{\sigma= \pm} 2^{1 / \nu} \frac{\xi T}{v}\left(\frac{r T}{2 v}\right)^{2 / \nu-2} \sinh ^{2 / \nu}\left(\frac{\pi \xi T}{v}\right) \\
& \times \exp \left(\frac{\omega+2 \sigma V}{2 T}\right) \\
& \times \mathcal{Q}\left(\frac{2 \pi \xi T}{v}, \frac{\omega+2 \sigma V}{\pi T}\right),
\end{aligned}
$$

where $\mathcal{Q}(a, b)$ is given in Eq. (24) and the normalization factor is given in Eq. (23). Taking this equation at $T \xi / v \ll 1$ one can show that the differential shot noise at zero bias is proportional to the conductance, namely, $\partial S_{d c}(0, V) / \partial V \propto$ $(r T / 2 v)^{2 / \nu-2}(\pi \xi T / v)^{2 / \nu}$. This expression vanishes at $T \rightarrow$ 0 . In particular we find that at zero temperature, zero frequency, $\omega=0$, and $V \xi / v \ll 1$ we obtain the asymptotic expression for shot noise:

$$
S_{d c}(0, V) / \tilde{I}_{0} \simeq \frac{4 \pi^{2}}{\Gamma[4 / \nu]} \frac{v \xi}{r^{2} V}\left(\frac{2 r \xi V^{2}}{\pi v^{2}}\right)^{2 / v}
$$

This expression results in vanishing differential shot noise, namely, $\partial S_{d c}(0, V) / \partial V=0$ at $V \rightarrow 0$. It is worth mentioning that at $v=1$ the result of this section agrees with the expressions of the previous section and Eq. (33) for Fano factor at finite temperatures is satisfied. We note that no resonances (singularities) appear in Eqs. (31), (35), or (37). For instance, at zero temperature, this can be seen from the fact that the power-law correlation functions result in the linear frequency behavior of noise $S(\omega, V) \propto \omega$ at small, $\omega \ll 2 V$, and large, $\omega \gg 2 V$, frequencies.

\section{TUNNELING CURRENT AND FINITE-FREQUENCY NOISE IN THE AC REGIME}

To study the case of time-dependent voltage, we assume a periodic bias of the form $\tilde{V}(t)=V+V_{1} \cos (\Omega t)$, where $\Omega$ is the driving frequency. The time-dependent part of such bias averages to zero over one period $\mathcal{T}=2 \pi / \Omega$, and the dc part of the time-averaged tunneling current in the case of ac bias is given by

$$
I=\frac{2}{\mathcal{T}} \int_{0}^{\mathcal{T}} d t \int_{-\infty}^{t} d t^{\prime} \operatorname{Re}\left\{e^{i \int_{t^{\prime}}^{t} \tilde{V}\left(t^{\prime}\right)}\left\langle\left[\hat{A}^{\dagger}(t), \hat{A}\left(t^{\prime}\right)\right]\right\rangle_{0}\right\} .
$$

With the exact form of the vertex operators from Appendixes $\mathrm{B}$ and $\mathrm{C}$, and using an expansion in terms of Bessel functions, $\exp [i \lambda \sin \varphi]=\sum_{n=-\infty}^{\infty} J_{n}(\lambda) \exp [\operatorname{in} \varphi]$, we find

$$
I=\sum_{n=-\infty}^{+\infty} J_{n}^{2}\left(2 V_{1} / \Omega\right) I_{d c}(V+n \Omega / 2),
$$

where $I_{d c}(V+n \Omega / 2)$ has been calculated in Eqs. (10), (14), (21), and (23). At $\Omega \rightarrow 0$ and $V_{1} \rightarrow 0$, the sum of Floquet factors goes to one, i.e., $\sum_{n=-\infty}^{\infty} J_{n}^{2}\left(2 V_{1} / \Omega\right) \rightarrow 1$, and thus we recover the result of Eq. (8) in the case of dc bias for all filling factors.

We proceed by calculating the noise in the presence of such an ac bias voltage. Again, we consider the finite-frequency noise averaged over a drive period $\mathcal{T}$. Due to the drive, this can be regarded as noise due to photon-assisted electron transport across the QPC. The time-averaged photon-assisted finite-frequency noise is given by the following Wigner transformation:

$$
S(\omega)=\frac{1}{\mathcal{T}} \int_{0}^{\mathcal{T}} d \tau \int_{-\infty}^{+\infty} d \tau^{\prime} S\left(\tau+\tau^{\prime} / 2, \tau-\tau^{\prime} / 2\right) e^{i \omega \tau^{\prime}}
$$

where we have introduced the "center of mass" and "relative" time variables, $\tau=\left(t+t^{\prime}\right) / 2$ and $\tau^{\prime}=t-t^{\prime}$, respectively. The integrand includes the current-current correlation function $S\left(t, t^{\prime}\right)=\left\langle\delta \hat{J}(t) \delta \hat{J}\left(t^{\prime}\right)\right\rangle$ with $\delta \hat{J}(t)=\hat{J}(t)-\langle\hat{J}(t)\rangle$ and the average is performed with respect to the biased ground state of the system. Using again an expansion of the exponent in terms of Bessel functions, the time invariance of the vertex correlation functions (see Appendix C) and $J_{-n}^{2}(x)=J_{n}^{2}(x)$ we get the final result for finite-frequency noise,

$$
S(\omega)=\sum_{n=-\infty}^{\infty} J_{n}^{2}\left(2 V_{1} / \Omega\right) S_{d c}(\omega, V+n \Omega / 2),
$$

where $S_{d c}(\omega, V+n \Omega / 2)$ has been calculated in Eqs. (30), (31), (35), and (37). Here again, as $\Omega \rightarrow 0$ and $V_{1} \rightarrow 0$, the sum of Floquet factors goes to one, i.e., 


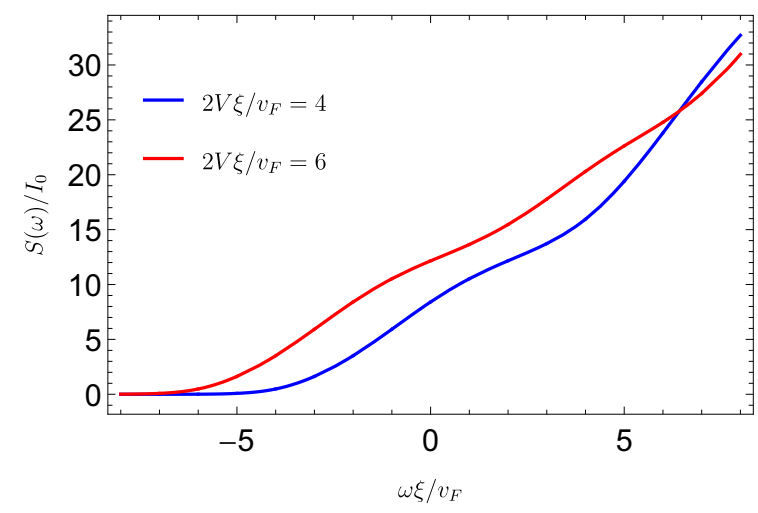

FIG. 6. The normalized finite-frequency nonsymmetrized noise of the tunneling current in the presence of periodic time-dependent (ac) bias, $\tilde{V}(t)=V+V_{1} \cos (\Omega t)$ at zero temperature for different $2 V \xi / v_{F}$ [see Eq. (42)]. Again we find no resonances. In this figure we show the case of filling factor $v=1$ and choose $S_{d c}(\omega, V)$ from Eq. (31). We set $p=0$ or $1,2 V_{1} \xi / v_{F}=2$, and $\Omega \xi / v_{F}=1$.

$\sum_{n=-\infty}^{\infty} J_{n}^{2}\left(2 V_{1} / \Omega\right) \rightarrow 1$, and thus we recover the result of Eq. (27) in the case of dc bias. The dependence of noise on frequency, Eq. (42) at filling factor $v=1$, is presented in Fig. 6. As in the case of dc bias, there are no resonances (singularities) in the frequency-dependent noise, which is a consequence of the positive power-law behavior with respect to frequency (see the discussion in the last paragraph of Sec. IV). In Ref. [95], in the low-frequency regime, it was experimentally demonstrated that a similar equation, $\Lambda=\sum_{n=-\infty}^{n=\infty} J_{n}^{2}\left(V_{1} / \Omega\right) \Lambda_{d c}(V+n \Omega)$, holds for electron current, heat current, and shot noise under ac bias over a QPC contact between two edge states. According to Eq. (42), this statement holds in one more general case, namely, for finite-frequency noise. This result can be used to interpret the experiments on dynamical response of Laughlin anyons in the presence of time-dependent bias [95].

\section{CONCLUSION}

In this paper we have studied tunneling between a superconductor and a $\mathrm{QH}$ edge state at different filling factors, namely, $v=1, v=2$, and $v=1 /(2 n+1)$. To account for electron-electron interaction in the $\mathrm{QH}$ edge state, we used a low-energy effective theory based on bosonization. In the bosonic picture of collective excitations, the spectrum splits into two modes, namely, the fast charge mode and slow dipole mode. Exact diagonalization allows us to calculate the twoand four-point equilibrium correlation functions, which are necessary to evaluate the transport properties of system, such as current and noise. We investigated the tunneling between the $\mathrm{QH}$ edge states and the superconductor to the lowest order in the tunneling coupling under the dc and ac biases.

For filling factor $v=1$, at zero temperature and $V \xi / v_{F} \ll$ 1 , we found that the tunneling current is proportional to $I_{d c}(V) \propto(\tau \Delta)^{2} \xi^{2} V^{3} / v_{F}^{4}$, which is a manifestation of nonOhmic behavior. This scaling of the tunneling current with the applied dc bias results in a vanishing conductance. At finite temperatures, at $\xi T / v_{F} \ll 1$ and $V / T \ll 1$, the current is proportional to the applied bias, and the density of states is renormalized by the dimensionless factor $\xi T / v_{F}$, namely, $I_{d c}(V) \propto\left(\tau \Delta / v_{F}\right)^{2}\left(\xi T / v_{F}\right)^{2} V$. In addition to the tunneling current, we presented results for the finite-frequency current noise. The ratio between shot noise and tunneling current, known as the Fano factor, was found to be $S_{d c}(0, V) / I_{d c}(V)=2$. Thus, the differential shot noise, $\partial S_{d c}(0, V) / \partial V$ at $V \rightarrow 0$, vanishes as well. At finite temperatures the Fano factor has the form $S_{d c}(0, V) / I_{d c}(V)=$ $2 \operatorname{coth}(V / T)$. As a result, at $\xi T / v_{F} \ll 1$, the differential shot noise at $V \rightarrow 0$ is proportional to the conductance and vanishes as $T \rightarrow 0$.

For filling factor $v=2$, in the case of simultaneous tunneling of a Cooper pair into different $\mathrm{QH}$ channels, the situation changes drastically. At zero temperature and $V \xi / v_{F} \ll 1$, the current manifests Ohmic behavior to leading order, $I_{d c}(V) \propto$ $\left(\tau \Delta / v_{F}\right)^{2}(1-\mathcal{N}) V$, where $0<\mathcal{N}<1$ in the case of simultaneous tunneling into two edge states. The shot noise is proportional to the current, $S_{d c}(0, V) / I_{d c}(V)=2$, so the differential shot noise at $V \rightarrow 0$ is generally not equal to zero. At low temperatures $\xi T / v_{F} \ll 1$ the leading behavior of both the conductance and the differential shot noise does not depend on temperature. The temperature dependence appears only in a subleading correction proportional to $\mathcal{N}\left(\xi T / v_{F}\right)^{2}$. In the presence of electron-electron interaction the results are qualitatively similar, but one has to replace the Fermi velocity by the geometric average of the velocities of the charged and dipole modes, $\sqrt{v_{1} v_{2}}$.

For filling factor $v=1 /(2 n+1)$, the power-law behavior of transport quantities depends on $v$. At $V \xi / v \ll 1$, the current is given by $I_{d c}(V) \propto V^{4 / v-1}$ and the conductance vanishes. At low temperatures, $\xi T / v \ll 1$, we have $I_{d c}(V) \propto$ $T^{4 / v-2} V$ and the conductance depends on temperature. The behavior of the differential shot noise at $V \rightarrow 0$ with respect to temperature is identical to that of the conductance, namely, $\propto T^{4 / v-2}$.

We also provided a general expression for the tunneling current and the finite-frequency noise in the presence of a periodic ac bias voltage. This result, valid for all filling factors considered, demonstrates that the current and finite-frequency noise can be expressed as the sum of dc currents and noise terms with Floquet coefficients. Recently, it was experimentally found that an expression similar to our result (42) holds for shot noise [95]. We have found that this statement holds in the more general case of finite-frequency noise.

At Laughlin filling factors, in addition to the Coulomb blockade [96], it has been found that the vanishing conductance [61] and differential shot noise at low temperatures is a consequence of an additional suppression mechanism called the Pauli blockade: after the tunneling of the first electron of a Cooper pair the tunneling of the second electron into the $\mathrm{QH}$ edge state is suppressed up to times $\xi / v_{F}$, where $v_{F}$ is the velocity of the edge excitations, due to the Pauli exclusion principle. At filling factor $v=2$, in the case of simultaneous tunneling of a Cooper pair into both channels, the Pauli blockade is partially removed. Electron-electron interactions do not change the physics qualitatively but result in a renormalization of the Fermi velocity. Finally, as a future perspective, it would be interesting to consider a similar problem in the context of levitonic physics [9], where the injection of single particles due to tailored voltage pulses is investigated. 


\section{ACKNOWLEDGMENTS}

We are grateful to Christian Glattli for fruitful discussions. The authors acknowledge financial support from the National Research Fund Luxembourg under Grants No. CORE C19/MS/13579612/HYBMES and No. ATTRACT 7556175.

\section{APPENDIX A: TWO-POINT CORRELATION FUNCTION}

In this Appendix we calculate the two-point correlation function of right-moving fermions, $G_{j}\left(x, t ; x^{\prime}, t^{\prime}\right)$, at filling factor $v=1$ and at finite temperature $T$. We use the bosonization technique, which is further necessary to take into account the electron-electron interaction. Here the subscript $j=1,2$ denotes the $\mathrm{QH}$ channel. According to the bosonization technique, we can write

$$
\begin{aligned}
G_{j}\left(x, x^{\prime} ; t, t^{\prime}\right) & =\left\langle\hat{\psi}_{j}^{\dagger}(x, t) \hat{\psi}_{j}\left(x^{\prime}, t^{\prime}\right)\right\rangle_{0} \\
& =\frac{1}{r}\left\langle e^{-i \hat{\phi}_{j}(x, t)} e^{i \hat{\phi}_{j}\left(x^{\prime}, t^{\prime}\right)}\right\rangle_{0}=\frac{1}{r} e^{M\left(x, t ; x^{\prime}, t^{\prime}\right)},
\end{aligned}
$$

where $r$ is an ultraviolet cutoff, and in Gaussian approximation under consideration the exponent is given by

$$
\begin{aligned}
M\left(x, t ; x^{\prime}, t^{\prime}\right)= & -\frac{1}{2}\left\langle\hat{\phi}_{j}^{2}(x, t)\right\rangle_{0}-\frac{1}{2}\left\langle\hat{\phi}_{j}^{2}\left(x^{\prime}, t^{\prime}\right)\right\rangle_{0} \\
& +\left\langle\hat{\phi}_{j}(x, t) \hat{\phi}_{j}\left(x^{\prime}, t^{\prime}\right)\right\rangle_{0} .
\end{aligned}
$$

Using the expansion of bosonic field in terms of creation and annihilation operators of bosons, we get the following expression (zero modes are ignored):

$$
\begin{aligned}
M\left(x, t ; x^{\prime}, t^{\prime}\right)= & \int_{0}^{\infty} \frac{d k}{k} e^{-\frac{r k}{2 \pi}}\left[\left(1+f_{B}(k)\right)\left(e^{i k\left[X(t)-X^{\prime}\left(t^{\prime}\right)\right]}-1\right)\right. \\
& \left.+f_{B}(k)\left(e^{-i k\left[X(t)-X^{\prime}\left(t^{\prime}\right)\right]}-1\right)\right],
\end{aligned}
$$

where $X=x-v_{j} t, X^{\prime}=x^{\prime}-v_{j} t^{\prime}$, and $f_{B}(k)=\left(e^{v_{j} k / T}-\right.$ $1)^{-1}=\sum_{n=1}^{\infty} e^{-v_{j} \beta k n}$ is an equilibrium bosonic distribution function with inverse temperature $\beta=1 / T$. Further integration with respect to momentum variable $k$ gives

$$
M\left(x, t ; x^{\prime}, t^{\prime}\right)=\log \left[\frac{i r / 2 \pi}{X(t)-X^{\prime}\left(t^{\prime}\right)+i r / 2 \pi}\right]-\sum_{n=1}^{\infty} \log \left[1+\frac{\left[\pi\left(X(t)-X^{\prime}\left(t^{\prime}\right)+i r / 2 \pi\right) / v_{j} \beta\right]^{2}}{\pi^{2} n^{2}}\right] .
$$

Next, exponentiating the above relation and using the definition of hyperbolic sine

$$
\sinh (z)=z \prod_{n=1}^{\infty}\left(1+\frac{z^{2}}{\pi^{2} n^{2}}\right)
$$

we finally get the result for the two-point correlation function at finite temperature:

$$
\begin{aligned}
G_{j}\left(x, x^{\prime} ; t, t^{\prime}\right) & =\frac{-i T}{2 v_{j}} \frac{1}{\sinh \left[\pi T\left(t-t^{\prime}-\left(x-x^{\prime}\right) / v_{j}-i \gamma\right)\right]}, \\
\gamma & \rightarrow+0 .
\end{aligned}
$$

The correlation function at zero temperature is obtained, using that $\sinh (x) \sim x$, namely,

$$
G_{j}\left(x, x^{\prime} ; t, t^{\prime}\right)=\frac{-i}{2 \pi v_{j}} \frac{1}{t-t^{\prime}-\left(x-x^{\prime}\right) / v_{j}-i \gamma} .
$$

\section{APPENDIX B: FOUR-POINT CORRELATION FUNCTION}

In this Appendix we derive the expression for the fourpoint correlation function [97]. We again use the Gaussian character of theory to calculate it; namely, the average of four vertex operators is written as the exponent of a combination of averages of bosonic field. To demonstrate this, we use the bosonization technique to rewrite the four-point correlation function, namely,

$$
\begin{aligned}
\mathcal{L}_{1} & =\left\langle\hat{\psi}_{1}^{\dagger}\left(x_{1}, t_{1}\right) \hat{\psi}_{1}^{\dagger}\left(x_{2}, t_{2}\right) \hat{\psi}_{1}\left(x_{3}, t_{3}\right) \hat{\psi}_{1}\left(x_{4}, t_{4}\right)\right\rangle_{0} \\
& =\frac{1}{r^{2}}\left\langle e^{-i \hat{\phi}_{1}\left(x_{1}, t_{1}\right)} e^{-i \hat{\phi}_{1}\left(x_{2}, t_{2}\right)} e^{i \hat{\phi}_{1}\left(x_{3}, t_{3}\right)} e^{i \hat{\phi}_{1}\left(x_{4}, t_{4}\right)}\right\rangle_{0},
\end{aligned}
$$

where we have omitted the arguments of $\mathcal{L}_{1}$ and the average is taken with respect to the equilibrium zero density matrix, $\hat{\rho}_{0}$. Next, using Eq. (19) from the main text, the above expression can be rewritten as a product of two four-point vertex correlation functions corresponding to charged and dipole modes in presence of interaction, namely, $\mathcal{L}_{1}=\mathcal{L}_{1}^{1} \times \mathcal{L}_{2}^{2}$, where $\hat{\chi}_{j}(x, t)=\hat{\chi}_{1}\left(x-v_{j} t\right)$ and consequently

$\mathcal{L}_{1}^{j}=\frac{1}{r}\left\langle e^{\frac{-i}{\sqrt{2}} \hat{\chi}_{j}\left(x_{1}-v_{j} t_{1}\right)} e^{\frac{-i}{\sqrt{2}} \hat{\chi}_{j}\left(x_{2}-v_{j} t_{2}\right)} e^{\frac{i}{\sqrt{2}} \hat{\chi}_{j}\left(x_{3}-v_{j} t_{3}\right)} e^{\frac{i}{\sqrt{2}} \hat{\chi}_{j}\left(x_{4}-v_{j} t_{4}\right)}\right\rangle_{0}$.

Further in Gaussian approximation [97], in terms of new bosonic fields, the above correlation function takes the form

$$
\begin{aligned}
\mathcal{L}_{1}^{j}= & \frac{1}{r} \exp \left[-\frac{1}{4} \sum_{i=1}^{4} \lambda_{i}^{2}\left\langle\hat{\chi}_{j}^{2}\left(x_{i}-v_{j} t_{i}\right)\right\rangle_{0}\right. \\
& \left.-\frac{1}{2} \sum_{i<l}^{4} \lambda_{i} \lambda_{l}\left\langle\hat{\chi}_{j}\left(x_{i}-v_{j} t_{i}\right) \hat{\chi}_{j}\left(x_{l}-v_{j} t_{l}\right)\right\rangle_{0}\right], \\
\lambda_{i}= & \pm 1 .
\end{aligned}
$$

Further calculations give the final result for correlation function

$$
\begin{aligned}
\mathcal{L}_{1}^{j} & =\frac{1}{r} \sqrt{\frac{L_{13} L_{14} L_{23} L_{24}}{L_{12} L_{34}}}, \\
L_{i j} & =\frac{-i r T}{2 v_{j}} \frac{1}{\sinh \left[\pi T\left(t_{i}-t_{j}-\left(x_{i}-x_{j}\right) / v_{j}-i \gamma\right)\right]}, \\
i, j & =1,2,3,4 .
\end{aligned}
$$

All other four-point correlation functions can be calculated in the same manner. The correlation function at zero temperature can be obtained using Eq. (A7). It is worth mentioning that the higher-order correlation functions in the perturbative expansion, which give the subleading corrections to current and noise, may depend on the regularization of the pointlike tunneling Hamiltonian [98]. 


\section{APPENDIX C: TIME INVARIANCE OF VERTEX CORRELATION FUNCTIONS}

In this Appendix we show the time invariance of vertex correlation functions, namely that

$$
\left\langle\hat{A}^{\dagger}(t) \hat{A}(0)\right\rangle_{0}=\left\langle\hat{A}(t) \hat{A}^{\dagger}(0)\right\rangle_{0}=\left\langle\hat{A}^{\dagger}(0) \hat{A}(-t)\right\rangle_{0} .
$$

To do this, we represent these vertex correlation functions though the two-point correlation functions $G_{j}\left(x-x^{\prime}, t-t^{\prime}\right)$ defined in Appendix B. For filling factor $v=1$ it is obvious because of Wick's theorem. For filling factor $v=2$ but without interaction

$$
\begin{aligned}
\left\langle\hat{A}^{\dagger}\left(t_{1}\right) \hat{A}\left(t_{2}\right)\right\rangle_{0}= & \left\langle\hat{A}\left(t_{1}\right) \hat{A}^{\dagger}\left(t_{2}\right)\right\rangle_{0}=\left[p^{2}+(1-p)^{2}\right] \sum_{j=1,2}\left[G_{j}\left(0, t_{1}-t_{2}\right) G_{j}\left(0, t_{1}-t_{2}\right)-G_{j}\left(\xi, t_{1}-t_{2}\right) G_{j}\left(-\xi, t_{1}-t_{2}\right)\right] \\
& +p(1-p)\left[2 G_{1}\left(0, t_{1}-t_{2}\right) G_{2}\left(0, t_{1}-t_{2}\right)-G_{1}\left(\xi, t_{1}-t_{2}\right) G_{2}\left(-\xi, t_{1}-t_{2}\right)-G_{2}\left(\xi, t_{1}-t_{2}\right) G_{1}\left(-\xi, t_{1}-t_{2}\right)\right],
\end{aligned}
$$

thus is it straightforward to confirm the time-invariance relations. At filling factor $v=2$ in the presence of interaction using Eq. (B3) and the commutation relation $\left[\hat{\chi}_{\alpha}\left(x_{1}-v_{\alpha} t_{1}\right), \hat{\chi}_{\beta}\left(x_{2}-v_{\beta} t_{2}\right)\right]=i \pi \delta_{\alpha \beta} \operatorname{sgn}\left(x_{1}-x_{2}-v_{\alpha} t_{1}+v_{\beta} t_{2}\right)$ one can justify Eq. (C1).

[1] K. v. Klitzing, G. Dorda, and M. Pepper, Phys. Rev. Lett. 45, 494 (1980).

[2] D. C. Tsui, H. L. Stormer, and A. C. Gossard, Phys. Rev. Lett. 48, 1559 (1982).

[3] Z. F. Ezawa, Quantum Hall Effects, 3rd ed. (World Scientific, Singapore, 2013).

[4] R. C. Ashoori, H. L. Stormer, L. N. Pfeiffer, K. W. Baldwin, and K. West, Phys. Rev. B 45, 3894 (1992).

[5] N. Kumada, H. Kamata, and T. Fujisawa, Phys. Rev. B 84, 045314 (2011).

[6] S. Tewari, P. Roulleau, C. Grenier, F. Portier, A. Cavanna, U. Gennser, D. Mailly, and P. Roche, Phys. Rev. B 93, 035420 (2016).

[7] D. C. Glattli, in The Quantum Hall Effect (Birkhäuser, Basel, 2005), pp. 163-197.

[8] B. Roussel, C. Cabart, G. Fève, E. Thibierge, and P. Degiovanni, Phys. Status Solidi B 254, 1600621 (2017).

[9] D. C. Glattli and P. S. Roulleau, Phys. Status Solidi B 254, 1600650 (2017).

[10] M. Kataoka, J. D. Fletcher, and N. Johnson, Phys. Status Solidi B 254, 1600547 (2017).

[11] C. Bäuerle, D. C. Glattli, T. Meunier, F. Portier, P. Roche, P. Roulleau, S. Takada, and X. Waintal, Rep. Prog. Phys. 81, 056503 (2018).

[12] T. Krähenmann, S. G. Fischer, M. Roosli, T. Ihn, C. Reichl, W. Wegscheider, K. Ensslin, Y. Gefen, and Y. Meir, Nat. Commun. 10, 3915 (2019).

[13] J. D. Fletcher, N. Johnson, E. Locane, P. See, J. P. Griffiths, I. Farrer, D. A. Ritchie, P. W. Brouwer, V. Kashcheyevs, and M. Kataoka, Nat. Commun. 10, 5298 (2019).

[14] Z. Iftikhar, S. Jezouin, A. Anthore, U. Gennser, F. D. Parmentier, A. Cavanna, and F. Pierre, Nature (London) 526, 233 (2015).

[15] Z. Iftikhar, A. Anthore, S. Jezouin, F. D. Parmentier, Y. Jin, A. Cavanna, A. Ouerghi, U. Gennser, and F. Pierre, Nat. Commun. 7, 12908 (2016).

[16] M. Banerjee, M. Heiblum, A. Rosenblatt, Y. Oreg, D. E. Feldman, A. Stern, and V. Umansky, Nature (London) 545, 75 (2017).
[17] M. Banerjee, M. Heiblum, V. Umansky, D. E. Feldman, Y Oreg, and A. Stern, Nature (London) 559, 205 (2018).

[18] T. D. Moore and D. A. Williams, Phys. Rev. B 59, 7308 (1999).

[19] P. Rickhaus, M. Weiss, L. Marot, and C. Schönenberger, Nano Lett. 12, 1942 (2012).

[20] F. Amet, C. T. Ke, I. V. Borzenets, J. Wang, K. Watanabe, T. Taniguchi, R. S. Deacon, M. Yamamoto, Y. Bomze, S. Tarucha, and G. Finkelstein, Science 352, 966 (2016).

[21] G.-H. Park, M. Kim, K. Watanabe, T. Taniguchi, and H.-J. Lee, Sci. Rep. 7, 10953 (2017).

[22] G.-H. Lee, K.-F. Huang, D. K. Efetov, D. S. Wei, S. Hart, T. Taniguchi, K. Watanabe, A. Yacoby, and P. Kim, Nat. Phys. 13, 693 (2017).

[23] Y. Ji, Y. Chung, D. Sprinzak, M. Heiblum, D. Mahalu, and H. Shtrikman, Nature (London) 422, 415 (2003).

[24] F. E. Camino, W. Zhou, and V. J. Goldman, Phys. Rev. Lett. 95, 246802 (2005).

[25] I. Neder, M. Heiblum, Y. Levinson, D. Mahalu, and V. Umansky, Phys. Rev. Lett. 96, 016804 (2006).

[26] P.-A. Huynh, F. Portier, H. le Sueur, G. Faini, U. Gennser, D. Mailly, F. Pierre, W. Wegscheider, and P. Roche, Phys. Rev. Lett. 108, 256802 (2012).

[27] R. Bhattacharyya, M. Banerjee, M. Heiblum, D. Mahalu, and V. Umansky, Phys. Rev. Lett. 122, 246801 (2019).

[28] H. Duprez, E. Sivre, A. Anthore, A. Aassime, A. Cavanna, A. Ouerghi, U. Gennser, and F. Pierre, Phys. Rev. X 9, 021030 (2019).

[29] H. Duprez, E. Sivre, A. Anthore, A. Aassime, A. Cavanna, U. Gennser, and F. Pierre, Science 366, 1243 (2019).

[30] S. Jezouin, Z. Iftikhar, A. Anthore, F. D. Parmentier, U. Gennser, A. Cavanna, A. Ouerghi, I. P. Levkivskyi, E. Idrisov, E. V. Sukhorukov, L. I. Glazman, and F. Pierre, Nature (London) 536, 58 (2016).

[31] Z. Iftikhar, A. Anthore, A. K. Mitchell, F. D. Parmentier, U. Gennser, A. Ouerghi, A. Cavanna, C. Mora, P. Simon, and F. Pierre, Science 360, 1315 (2018).

[32] A. Anthore, Z. Iftikhar, E. Boulat, F. D. Parmentier, A. Cavanna, A. Ouerghi, U. Gennser, and F. Pierre, Phys. Rev. X 8, 031075 (2018). 
[33] E. Sivre, A. Anthore, F. D. Parmentier, A. Cavanna, U. Gennser, A. Ouerghi, Y. Jin, and F. Pierre, Nat. Phys. 14, 145 (2018).

[34] C. Altimiras, H. le Sueur, U. Gennser, A. Cavanna, D. Mailly, and F. Pierre, Nat. Phys. 6, 34 (2010).

[35] H. le Sueur, C. Altimiras, U. Gennser, A. Cavanna, D. Mailly, and F. Pierre, Phys. Rev. Lett. 105, 056803 (2010).

[36] C. Altimiras, H. le Sueur, U. Gennser, A. Cavanna, D. Mailly, and F. Pierre, Phys. Rev. Lett. 105, 226804 (2010).

[37] A. Grivnin, H. Inoue, Y. Ronen, Y. Baum, M. Heiblum, V. Umansky, and D. Mahalu, Phys. Rev. Lett. 113, 266803 (2014).

[38] K. Itoh, R. Nakazawa, T. Ota, M. Hashisaka, K. Muraki, and T. Fujisawa, Phys. Rev. Lett. 120, 197701 (2018).

[39] E. G. Idrisov, I. P. Levkivskyi, and E. V. Sukhorukov, Phys. Rev. B 101, 245426 (2020).

[40] E. Weisz, H. K. Choi, I. Sivan, M. Heiblum, Y. Gefen, D. Mahalu, and V. Umansky, Science 344, 1363 (2014).

[41] X.-G. Wen, Adv. Phys. 44, 405 (1995).

[42] X.-G. Wen, Phys. Rev. B 44, 5708 (1991).

[43] C. de C. Chamon, D. E. Freed, and X. G. Wen, Phys. Rev. B 51, 2363 (1995).

[44] C. de C. Chamon, D. E. Freed, and X. G. Wen, Phys. Rev. B 53, 4033 (1996).

[45] C. de C. Chamon and X. G. Wen, Phys. Rev. Lett. 70, 2605 (1993).

[46] C. L. Kane and M. P. A. Fisher, Phys. Rev. Lett. 72, 724 (1994).

[47] C. L. Kane and M. P. A. Fisher, Phys. Rev. B 67, 045307 (2003).

[48] A. Crépieux, P. Devillard, and T. Martin, Phys. Rev. B 69, 205302 (2004).

[49] I. Safi, P. Devillard, and T. Martin, Phys. Rev. Lett. 86, 4628 (2001).

[50] T. K. T. Nguyen, A. Crépieux, T. Jonckheere, A. V. Nguyen, Y. Levinson, and T. Martin, Phys. Rev. B 74, 153303 (2006).

[51] I. Safi, Phys. Rev. B 99, 045101 (2019).

[52] B. Roussel, P. Degiovanni, and I. Safi, Phys. Rev. B 93, 045102 (2016).

[53] O. Parlavecchio, C. Altimiras, J.-R. Souquet, P. Simon, I. Safi, P. Joyez, D. Vion, P. Roche, D. Esteve, and F. Portier, Phys. Rev. Lett. 114, 126801 (2015).

[54] I. Safi and E. V. Sukhorukov, Europhys. Lett. 91, 67008 (2010).

[55] D. Ferraro, M. Carrega, A. Braggio, and M. Sassetti, New J. Phys. 16, 043018 (2014).

[56] E. Bocquillon, V. Freulon, F. D. Parmentier, J.-M. Berroir, B. Plaçais, C. Wahl, J. Rech, T. Jonckheere, T. Martin, C. Grenier, D. Ferraro, P. Degiovanni, and G. Fève, Ann. Phys. 526, 1 (2014).

[57] J. Rech, D. Ferraro, T. Jonckheere, L. Vannucci, M. Sassetti, and T. Martin, Phys. Rev. Lett. 118, 076801 (2017).

[58] D. Ferraro, J. Rech, T. Jonckheere, and T. Martin, Phys. Rev. B 91, 075406 (2015).

[59] M. Acciai, A. Calzona, G. Dolcetto, T. L. Schmidt, and M. Sassetti, Phys. Rev. B 96, 075144 (2017).

[60] G. Dolcetto and T. L. Schmidt, Phys. Rev. B 94, 075444 (2016).

[61] M. P. A. Fisher, Phys. Rev. B 49, 14550 (1994).

[62] D. L. Maslov, M. Stone, P. M. Goldbart, and D. Loss, Phys. Rev. B 53, 1548 (1996).
[63] E.-A. Kim, S. Vishveshwara, and E. Fradkin, Phys. Rev. Lett. 93, 266803 (2004).

[64] P. Virtanen and P. Recher, Phys. Rev. B 85, 035310 (2012).

[65] Z. Hou, Y. Xing, A.-M. Guo, and Q.-F. Sun, Phys. Rev. B 94, 064516 (2016).

[66] H. Hoppe, U. Zülicke, and G. Schön, Phys. Rev. Lett. 84, 1804 (2000).

[67] O. Gamayun, J. A. Hutasoit, and V. V. Cheianov, Phys. Rev. B 96, 241104(R) (2017).

[68] L. Cohnitz, A. De Martino, W. Häusler, and R. Egger, Phys. Rev. B 96, 140506(R) (2017).

[69] F. Giazotto, M. Governale, U. Zülicke, and F. Beltram, Phys. Rev. B 72, 054518 (2005).

[70] J. A. M. van Ostaay, A. R. Akhmerov, and C. W. J. Beenakker, Phys. Rev. B 83, 195441 (2011).

[71] N. M. Chtchelkatchev and I. S. Burmistrov, Phys. Rev. B 75, 214510 (2007).

[72] K. Komatsu, C. Li, S. Autier-Laurent, H. Bouchiat, and S. Guéron, Phys. Rev. B 86, 115412 (2012).

[73] S. Guiducci, M. Carrega, G. Biasiol, L. Sorba, F. Beltram, and S. Heun, Phys. Status Solidi (RRL) 13, 1800222 (2019).

[74] S. Guiducci, M. Carrega, F. Taddei, G. Biasiol, H. Courtois, F. Beltram, and S. Heun, Phys. Rev. B 99, 235419 (2019).

[75] N. H. Lindner, E. Berg, G. Refael, and A. Stern, Phys. Rev. X 2, 041002 (2012).

[76] D. J. Clarke, J. Alicea, and K. Shtengel, Nat. Commun. 4, 1348 (2013).

[77] J. Klinovaja and D. Loss, Phys. Rev. B 90, 045118 (2014).

[78] J. Alicea and P. Fendley, Annu. Rev. Condens. Matter Phys. 7, 119 (2016).

[79] C. J. Pedder, T. Meng, R. P. Tiwari, and T. L. Schmidt, Phys. Rev. B 96, 165429 (2017).

[80] A. Calzona, T. Meng, M. Sassetti, and T. L. Schmidt, Phys. Rev. B 98, 201110(R) (2018).

[81] T. Wu, Z. Wan, A. Kazakov, Y. Wang, G. Simion, J. Liang, K. W. West, K. Baldwin, L. N. Pfeiffer, Y. Lyanda-Geller, and L. P. Rokhinson, Phys. Rev. B 97, 245304 (2018).

[82] S. Groenendijk, A. Calzona, H. Tschirhart, E. G. Idrisov, and T. L. Schmidt, Phys. Rev. B 100, 205424 (2019).

[83] T. L. Schmidt, Eur. Phys. J.: Spec. Top. 229, 621 (2020).

[84] N. Schiller, E. Cornfeld, E. Berg, and Y. Oreg, Phys. Rev. Research 2, 023296 (2020).

[85] A. M. Chang, C. R. Phys. 3, 677 (2002).

[86] T. Wakamura, N. Hasegawa, K. Ohnishi, Y. Niimi, and Y. C. Otani, Phys. Rev. Lett. 112, 036602 (2014).

[87] Y. Kim, J. Zhang, E. Rossi, and R. M. Lutchyn, Phys. Rev. Lett. 114, 236804 (2015).

[88] A. I. Larkin and A. A. Varlamov, Theory of Fluctuations in Superconductors (Oxford University Press, Oxford, 2005).

[89] A. O. Slobodeniuk, E. G. Idrisov, and E. V. Sukhorukov, Phys. Rev. B 93, 035421 (2016).

[90] E. G. Idrisov, I. P. Levkivskyi, and E. V. Sukhorukov, Phys. Rev. Lett. 121, 026802 (2018).

[91] E. G. Idrisov, Phys. Rev. B 100, 155422 (2019).

[92] T. Patlatiuk, C. P. Scheller, D. Hill, Y. Tserkovnyak, G. Barak, A. Yacoby, L. N. Pfeiffer, K. W. West, and D. M. Zumbühl, Nat. Commun. 9, 3692 (2018). 
[93] G. Lesovik and R. Loosen, JETP Lett. 65, 295 (1997).

[94] R. Zamoum, M. Lavagna, and A. Crépieux, J. Stat. Mech.: Theory Exp. (2016) 054013.

[95] M. Kapfer, P. Roulleau, M. Santin, I. Farrer, D. A. Ritchie, and D. C. Glattli, Science 363, 846 (2019).
[96] C. L. Kane and M. P. A. Fisher, Phys. Rev. B 46, 15233 (1992).

[97] E. G. Idrisov, I. P. Levkivskyi, and E. V. Sukhorukov, Phys. Rev. B 96, 155408 (2017).

[98] M. Filippone and P. W. Brouwer, Phys. Rev. B 94, 235426 (2016). 